\title{
Mixed Convection Flow over an Unsteady Stretching Surface in a Porous Medium with Heat Source
}

\author{
Syed Muhammad Imran, ${ }^{1}$ Saleem Asghar, ${ }^{1,2}$ \\ and Muhammad Mushtaq ${ }^{1}$ \\ ${ }^{1}$ Department of Mathematics, COMSATS Institute of Information Technology, Park Road, Chak Shahzad, \\ Islamabad 44000, Pakistan \\ ${ }^{2}$ Department of Mathematics, King Abdulaziz University, Jeddah 80200, Saudi Arabia \\ Correspondence should be addressed to Syed Muhammad Imran, syedsiim@gmail.com
}

Received 27 March 2012; Revised 22 September 2012; Accepted 26 September 2012

Academic Editor: Trung Nguyen Thoi

Copyright (C) 2012 Syed Muhammad Imran et al. This is an open access article distributed under the Creative Commons Attribution License, which permits unrestricted use, distribution, and reproduction in any medium, provided the original work is properly cited.

\begin{abstract}
This paper deals with the analysis of an unsteady mixed convection flow of a fluid saturated porous medium adjacent to heated/cooled semi-infinite stretching vertical sheet in the presence of heat source. The unsteadiness in the flow is caused by continuous stretching of the sheet and continuous increase in the surface temperature. We present the analytical and numerical solutions of the problem. The effects of emerging parameters on field quantities are examined and discussed.
\end{abstract}

\section{Introduction}

The study of flow and heat transfer over a continuous stretching sheet with a given temperature distribution has received much attention due to its applications in different fields of engineering and industry. The stretching and heating/cooling of the plate have a definite impact on the quality of the finished product. The modeling of the real processes is thus undertaken with the help of different stretching velocities and temperature distributions. Examples of such processes are the extrusion of polymers, aerodynamic extrusion of plastic sheets, and the condensation process of a metallic plate (cf. Altan et al. [1]; Fisher [2]). A few more examples of importance are heat-treated materials traveling between a feed roll and a wind-up roll or materials manufactured by extrusion, wire drawing, spinning of filaments, glass-fiber and paper production, cooling of metallic sheets or electronic chips, crystal growing, food processing, and so forth. A great deal of research in fluid mechanics is rightfully produced to model these problems and to provide analytical and numerical 
results for a better understanding of the fluid behavior and an adequate explanation of the experiments.

Sakiadis [3] was first to present the boundary layer flow on a continuous moving surface in a viscous medium. Crane [4] was first to obtain an analytical solution for the steady stretching of the surface for viscous fluid. The heat transfer analysis for a stretching surface was studied by Erickson et al. [5], while heat and mass transfer for stretching surfaces was addressed by P. S. Gupta and A. S. Gupta [6]. Some of the research pertaining to the steady stretching is given in numerous references [7-16]. In these discussions, steady state stretching and heat transfer analyses have been undertaken.

In some cases the flow and heat transfer can be unsteady due to a sudden or oscillating stretching of the plates or by time-varying temperature distributions. Physically, it concerns the rate of cooling in the steady fabrication processes and the transient crossover to the steady state. These observations are generally investigated in the momentum and thermal boundary layer by assuming a steady part of the stretching velocity proportional to the distance from the edge and an unsteady part to the inverse of time (highlighting the cooling process). A similarity solution of the unsteady Navier-Stokes equations, of a thin liquid film on a stretching sheet, was considered by Wang [17]. Andersson et al. [18] extended this problem to heat transfer analysis for a power law fluid. Unsteady flow past a wall which starts to move impulsively has been presented byPop and Na [19]. The heat transfer characteristics of the flow problem of Wang [17] were considered by Andersson et al. [18]. The effect of the unsteadiness parameter on heat transfer and flow field over a stretching surface with and without heat generation was considered by [21, 22], respectively. The numerical solutions of the boundary layer flow and heat transfer over an unsteady stretching vertical surface were presented by Ishak et al. [23,24]. Some more works regarding unsteady stretching are reported and available [25-27].

It is sometimes physically interesting to examine the flow, thermal flow, and thermal characteristics of viscous fluids over a stretching sheet in a porous medium. For example, in the physical process of drawing a sheet from a slit of a container, it is tacitly assumed that only the fluid adhered to the sheet is moving but the porous matrix remains fixed to follow the usual assumption of fluid flow in a porous medium. Different models of the porous medium have been formulated, namely, the Darcy, Brinkman, Darcy Brinkman, and Forchheimer models. However, the Darcy Brinkman model is widely accepted as the most appropriate. Comprehensive reviews of the convection through a porous media have been addressed in the studies [28-35].

We all know that mixed convection is induced by the motion of a solid material (forced convection) and thermal buoyancy (natural convection). The buoyancy forces stemming from the heating or cooling of the continuous stretching sheets alter the flow and thermal fields and thereby the heat transfer characteristics of the manufacturing process. The combined forced and free convection in a boundary layer over continuous moving surfaces through an otherwise quiescent fluid have been investigated by many authors [36-43].

The introduction of a heat source/sink in the fluid is sometimes important because of sharp temperature distributions between solid boundaries and the ambient temperature that may influence the heat transfer analysis as reported by Vajravelu and Hadjinicolaou [44]. These sources can be generally space and temperature dependent.

Keeping in view the importance of all that has been previously stated and the progress still needed in these areas, we address the problem of an unsteady mixed convection flow in a fluid saturated porous medium adjacent to a heated/cooled semi-infinite stretching vertical sheet with a heat source. We present an analytical and numerical solution to attain an 


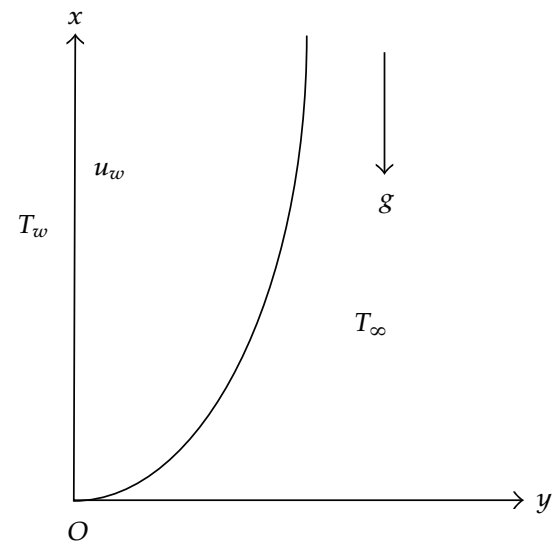

(a) Assisting flow

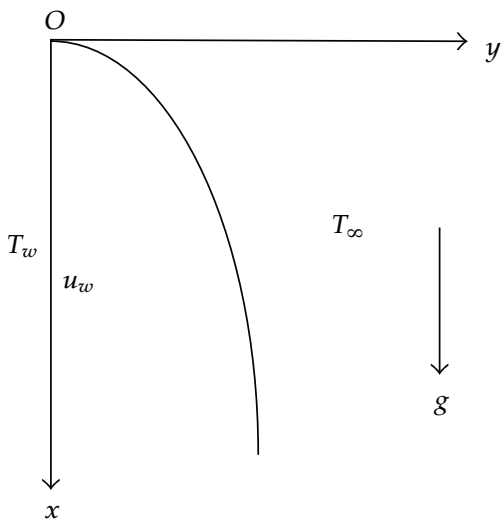

(b) Opposing flow

Figure 1: Physical model and coordinate system.

appropriate degree of confidence in both solutions. This paper has thus multiple objectives to meet. The presentation of a satisfactory analytical solution for unsteady stretching which can be used in future studies for unsteady problems, the introduction of a source/sink, and the consideration of a porous medium.

In mathematical terms, the governing coupled nonlinear differential equations are transformed into a nondimensional self-similar ordinary differential equation using the appropriate similarity variables. The transformed equations are then solved analytically and numerically using the perturbation method (with Padé approximation) and shooting method, respectively. Very good agreement has been seen. The effects of the emerging parameters are investigated on the field quantities with the help of graphs and the physical reasoning. A comparison is made with the existing literature to support the validity of our results.

\section{Development of the Flow Problem}

Consider an unsteady laminar mixed convection flow along a vertical stretched heated/cooled semi-infinite flat sheet. The sheet is assumed impermeable and immersed in a saturated porous medium satisfying the Darcy Brinkman model. At time $t=0$, the sheet is stretched with the velocity $u_{w}(x, t)$ and raised to temperature $T_{w}(x, t)$. The geometry of the problem is shown in Figure 1.

Under these assumptions, using the boundary layer and Boussinesq approximations, the unsteady two-dimensional Navier-Stokes equations and energy equation in the presence of heat source can be written as

$$
\frac{\partial u}{\partial x}+\frac{\partial v}{\partial y}=0,
$$




$$
\begin{gathered}
\frac{\partial u}{\partial t}+u \frac{\partial u}{\partial x}+v \frac{\partial u}{\partial y}=v \frac{\partial^{2} u}{\partial y^{2}}-\frac{v}{K} u+g \beta\left(T-T_{\infty}\right), \\
\frac{\partial T}{\partial t}+u \frac{\partial T}{\partial x}+v \frac{\partial T}{\partial y}=\alpha_{m} \frac{\partial^{2} T}{\partial y^{2}}+\frac{q^{\prime \prime \prime}}{\rho c_{p}}
\end{gathered}
$$

The appropriate boundary conditions of the problem are

$$
\begin{gathered}
u=u_{w}(x, t), \quad v=0, \quad T=T_{w}(x, t), \quad \text { at } y=0, \\
u \longrightarrow 0, \quad T \longrightarrow T_{\infty} \quad \text { as } y \longrightarrow \infty .
\end{gathered}
$$

In the above equations $u$ and $v$ are the velocity components in the $x$ and $y$ directions, respectively, $T$ is the fluid temperature inside the boundary layer, $K$ is the permeability of the porous medium, $t$ is time, $\alpha_{m}$ and $v$ are the thermal diffusivity and the kinematic viscosity, respectively. Where $q^{\prime \prime \prime}$ is the internal heat generation/absorption per unit volume. The value of $q^{\prime \prime \prime}$ is chosen as

$$
q^{\prime \prime \prime}=\frac{k_{m} u_{w}(x, t)}{x \mathcal{V}}\left[A^{*}\left(T_{w}-T_{\infty}\right)+B^{*}\left(T-T_{\infty}\right)\right]
$$

where $A^{*}$ and $B^{*}$ are space-dependent and temperature-dependent heat generation/absorption parameters and are positive for an internal heat source and negative for an internal heat sink. We assume that the stretching velocity $u_{w}(x, t)$ and the surface temperature $T_{w}(x, t)$ are

$$
\begin{gathered}
u_{w}(x, t)=\frac{a x}{1-c t^{\prime}} \\
T_{w}(x, t)=T_{\infty}+\frac{b x}{(1-c t)^{2}},
\end{gathered}
$$

where $a>0$ and $c>0$ are the constants having dimension time ${ }^{-1}$ such that $c t<1$. The constant $b$ has a dimension temperature/length, with $b>0$ and $b<0$ corresponding to the assisting and opposing flows, respectively, and $b=0$ is for a forced convection limit (absence of buoyancy force).

Let us introduce stream function $\psi$, similarity variable $\eta$ and nondimensional temperature $\theta$ as

$$
\begin{gathered}
\psi=\left(\frac{v a}{1-c t}\right)^{1 / 2} x f(\eta), \\
\theta(\eta)=\frac{T-T_{\infty}}{T_{w}-T_{\infty}}, \\
\eta=\left(\frac{a}{v(1-c t)}\right)^{1 / 2} y .
\end{gathered}
$$




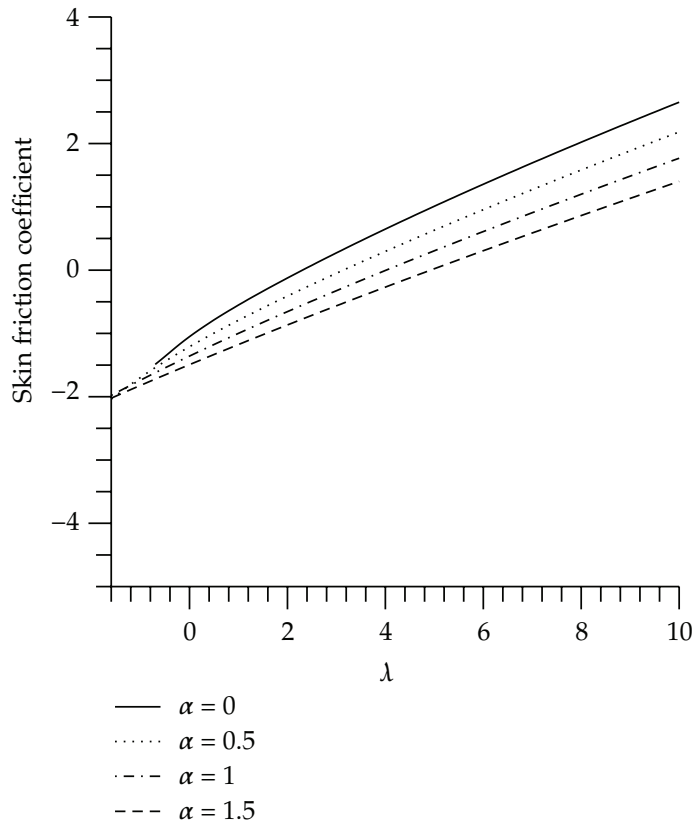

(a)

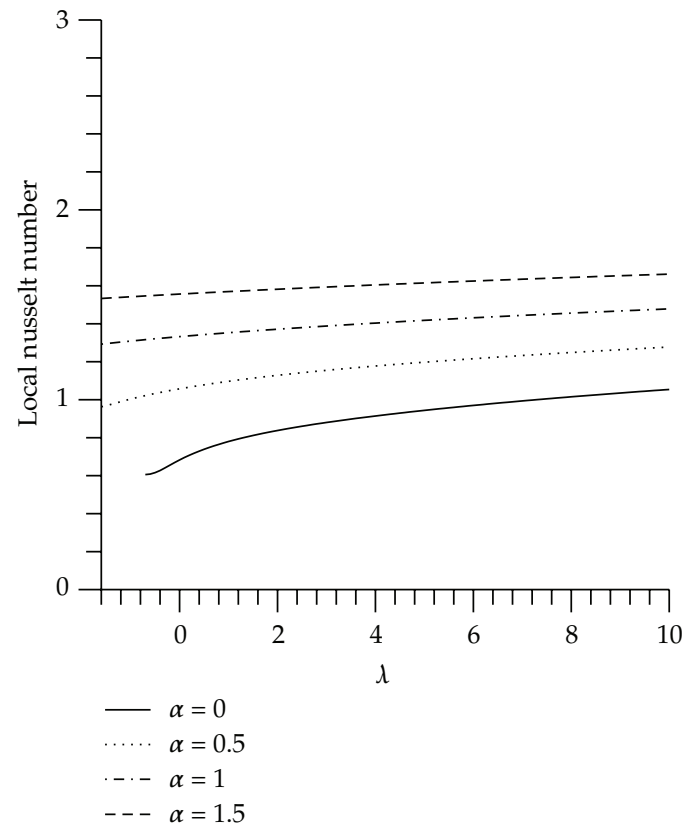

(b)

Figure 2: Variation of (a) skin friction coefficient (b) local Nusselt number with $\lambda$ for various values of unsteadiness parameter $\alpha$ when $\operatorname{Pr}=0.72, A^{*}=B^{*}=d=0.1$.

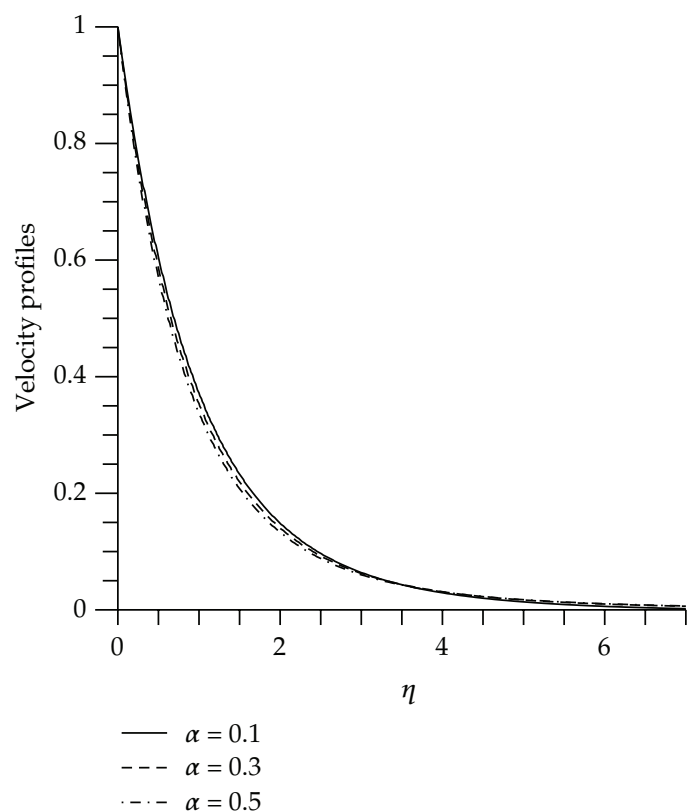

(a)

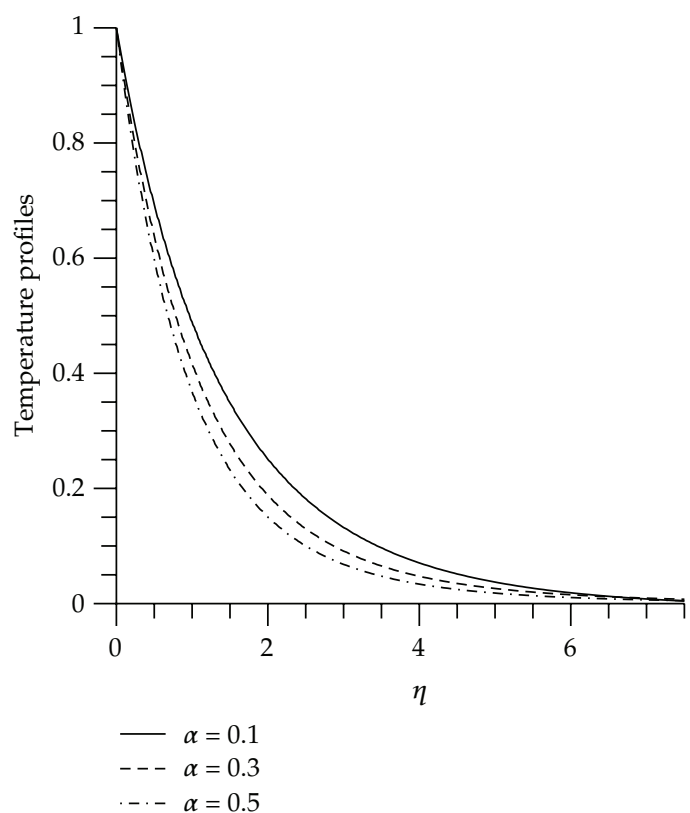

(b)

Figure 3: Effect of unsteadiness parameter $\alpha$ for the case of $\operatorname{Pr}=0.72, \lambda=d=A^{*}=B^{*}=0.1$ on (a) velocity distributions $f^{\prime}(\eta)(b)$ temperature distributions $\theta(\eta)$. 


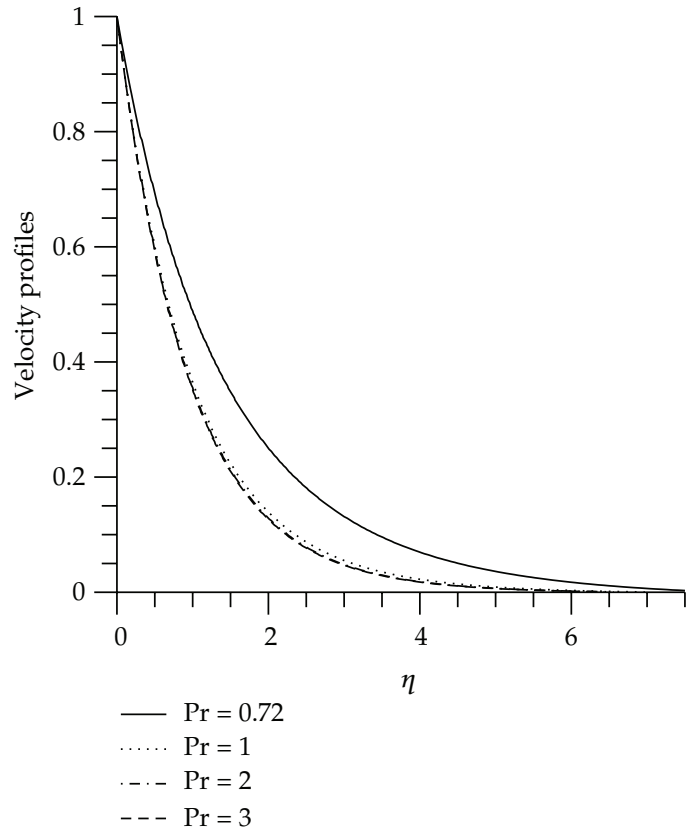

(a)

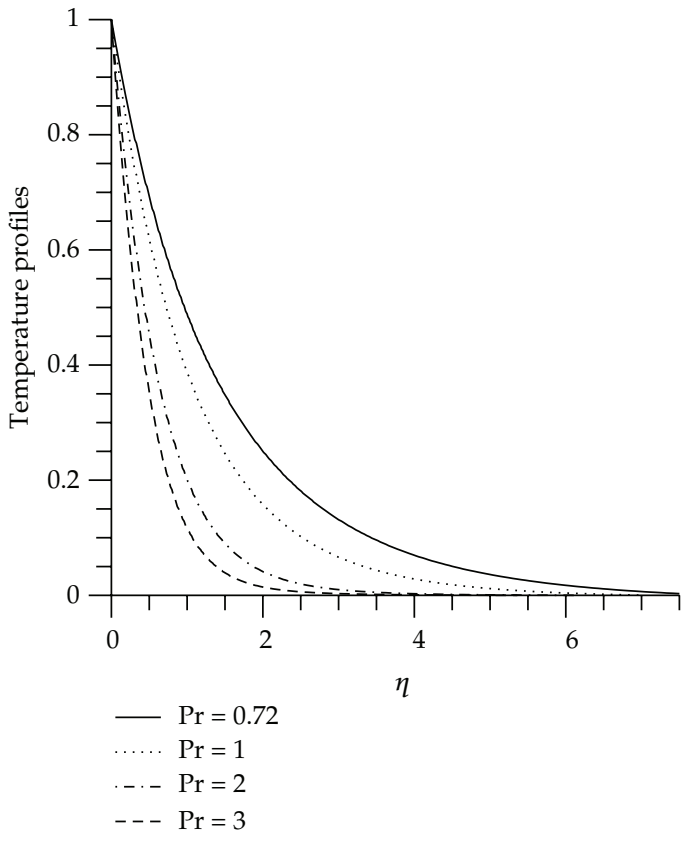

(b)

Figure 4: Effect of Prandtle number Pr for the case of $\alpha=\lambda=d=A^{*}=B^{*}=0.1$ on (a) velocity distributions $f^{\prime}(\eta)(b)$ temperature distributions $\theta(\eta)$.

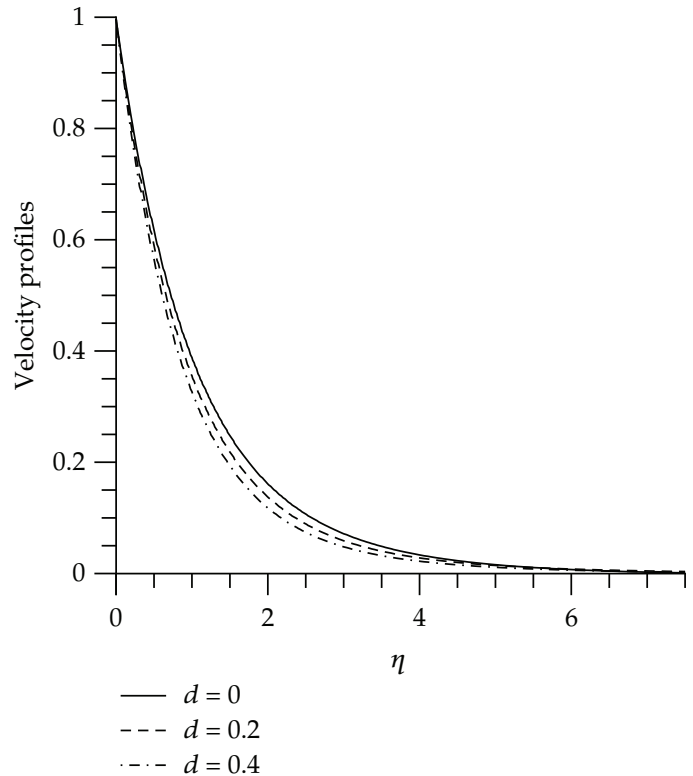

(a)

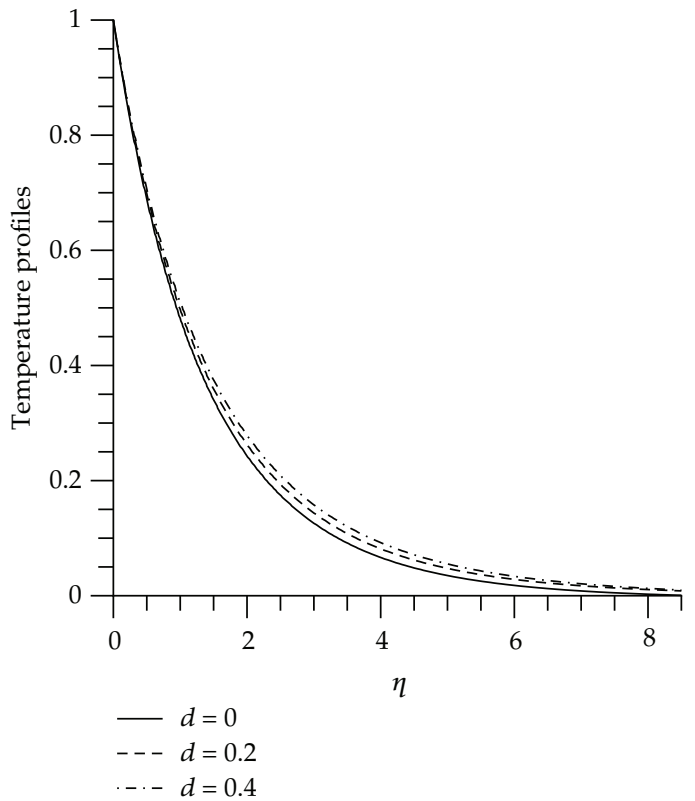

(b)

Figure 5: Effect of permeability parameter $d$ for the case of $\operatorname{Pr}=0.72, \alpha=\lambda=A^{*}=B^{*}=0.1$ on (a) velocity distributions $f^{\prime}(\eta)$, (b) temperature distributions $\theta(\eta)$. 
Table 1: Comparison between analytical and numerical results for $f^{\prime}(\eta)$ and $\theta(\eta)$ when $\operatorname{Pr}=0.72, d=$ $A^{*}=B^{*}=0.1$.

\begin{tabular}{|c|c|c|c|c|c|c|c|c|}
\hline \multirow{3}{*}{$\eta$} & \multicolumn{4}{|c|}{$f^{\prime}(\eta)$} & \multicolumn{4}{|c|}{$\theta(\eta)$} \\
\hline & \multicolumn{2}{|c|}{$\alpha=0$} & \multicolumn{2}{|c|}{$\alpha=0.1$} & \multicolumn{2}{|c|}{$\alpha=0$} & \multicolumn{2}{|c|}{$\alpha=0.1$} \\
\hline & Analytical & Numerical & Analytical & Numerical & Analytical & Numerical & Analytical & Numerical \\
\hline 0.0 & 1.00000 & 1.00000 & 1.00000 & 1.00000 & 1.00000 & 1.00000 & 1.00000 & 1.00000 \\
\hline 0.5 & 0.58647 & 0.58583 & 0.59846 & 0.59832 & 0.73927 & 0.74197 & 0.67861 & 0.68765 \\
\hline 1.0 & 0.34721 & 0.34589 & 0.36603 & 0.36717 & 0.56066 & 0.56613 & 0.46850 & 0.48402 \\
\hline 1.5 & 0.20676 & 0.20533 & 0.22722 & 0.23073 & 0.43085 & 0.43856 & 0.32808 & 0.34603 \\
\hline 2.0 & 0.12208 & 0.12092 & 0.14055 & 0.14680 & 0.33099 & 0.34017 & 0.23163 & 0.24820 \\
\hline 2.5 & 0.07283 & 0.07209 & 0.08792 & 0.09617 & 0.25591 & 0.26522 & 0.16806 & 0.18043 \\
\hline 3.0 & 0.04310 & 0.04278 & 0.05481 & 0.06386 & 0.19735 & 0.20634 & 0.12483 & 0.13127 \\
\hline 3.5 & 0.02497 & 0.02504 & 0.03385 & 0.04259 & 0.15122 & 0.15861 & 0.09493 & 0.09486 \\
\hline 4.0 & 0.01434 & 0.01473 & 0.02116 & 0.02883 & 0.11641 & 0.12151 & 0.07454 & 0.06867 \\
\hline 4.5 & 0.00827 & 0.00889 & 0.01368 & 0.01999 & 0.09113 & 0.09373 & 0.06048 & 0.05029 \\
\hline 5.0 & 0.00425 & 0.00507 & 0.00855 & 0.01326 & 0.06980 & 0.06959 & 0.04864 & 0.03517 \\
\hline 5.5 & 0.00208 & 0.00305 & 0.00570 & 0.00904 & 0.05524 & 0.05269 & 0.04025 & 0.02503 \\
\hline 6.0 & 0.00065 & 0.00175 & 0.00374 & 0.00575 & 0.00575 & 0.03836 & 0.03288 & 0.01669 \\
\hline
\end{tabular}

Table 2: Values of $-\theta^{\prime}(0)$ when $\alpha=\lambda=d=A^{*}=B^{*}=0$ and comparison with previous work.

\begin{tabular}{ccccccc}
\hline $\operatorname{Pr}$ & Grubka and Bobba [7] & \multirow{2}{*}{ Ali [9] } & Elbashbeshy [11] & Ishak et al. [24] & \multicolumn{2}{c}{ Present study } \\
& & & & & & \\
Numerical & Analytical \\
\hline 0.72 & 0.8086 & 0.8058 & 0.8161 & 0.8086 & 0.8086 & 0.8086 \\
1.00 & 1.0000 & 0.9961 & 1.0000 & 1.0000 & 1.0000 & 1.0000 \\
3.00 & 1.9237 & 1.9144 & & 1.9237 & 1.9237 & 1.9234 \\
10.0 & 3.7207 & 3.7206 & 3.7202 & 3.7207 & 3.7207 & 3.7205 \\
\hline
\end{tabular}

The velocity components are defined by

$$
u=\frac{\partial \psi}{\partial y}, \quad v=-\frac{\partial \psi}{\partial x}
$$

Substituting (2.7) into (2.2) and (2.3) we obtain

$$
\begin{gathered}
f^{\prime \prime \prime}+f f^{\prime \prime}-f^{\prime 2}-\alpha\left(f^{\prime}+\frac{1}{2} \eta f^{\prime \prime}\right)+\lambda \theta-d f^{\prime}=0, \\
\frac{1}{p r}\left(\theta^{\prime \prime}+A^{*} f^{\prime}+B^{*} \theta\right)+f \theta^{\prime}-f^{\prime} \theta-\alpha\left(2 \theta+\frac{1}{2} \eta \theta^{\prime}\right)=0,
\end{gathered}
$$

together with the boundary conditions

$$
\begin{gathered}
f(0)=0, \quad f^{\prime}(0)=1, \quad \theta(0)=1, \\
f^{\prime}(\infty)=0, \quad \theta(\infty)=0,
\end{gathered}
$$


Table 3: The values of $f^{\prime \prime}(0)$ for various values of $d$ and $\operatorname{Pr}$ when $\alpha=0, \lambda=1, A^{*}=B^{*}=0$.

\begin{tabular}{|c|c|c|c|c|c|c|c|c|}
\hline \multirow{2}{*}{$d$} & \multicolumn{4}{|c|}{ Results of Ishak et al. [23] } & \multicolumn{4}{|c|}{ Results of present study } \\
\hline & $\operatorname{Pr}=0.72$ & $\operatorname{Pr}=1.0$ & $\operatorname{Pr}=10$ & $\operatorname{Pr}=100$ & $\operatorname{Pr}=0.72$ & $\operatorname{Pr}=1.0$ & $\operatorname{Pr}=10$ & $\operatorname{Pr}=100$ \\
\hline 0.1 & -0.5631 & -0.6110 & -0.8743 & -0.9887 & -0.5631 & -0.6109 & -0.8744 & -0.9882 \\
\hline 1.0 & -0.9625 & -1.0000 & -1.2404 & -1.3541 & -0.9626 & -1.0000 & -1.2404 & -1.3543 \\
\hline 3.0 & -1.6163 & -1.6397 & -1.8307 & -1.9403 & -1.6166 & -1.6398 & -1.8307 & -1.9404 \\
\hline 5.0 & -2.1118 & -2.1281 & -2.2848 & -2.3902 & -2.1125 & -2.1286 & -2.2848 & -2.3903 \\
\hline
\end{tabular}

Table 4: The values of $-\theta^{\prime}(0)$ for various values of $d$ and $\operatorname{Pr}$ when $\alpha=0, \lambda=1, A^{*}=B^{*}=0$.

\begin{tabular}{lcccccccc}
\hline \multirow{2}{*}{ Results of Ishak et al. [23] } & \multicolumn{4}{c}{ Results of present study } \\
& $\operatorname{Pr}=0.72$ & $\operatorname{Pr}=1.0$ & $\operatorname{Pr}=10$ & $\operatorname{Pr}=100$ & $\operatorname{Pr}=0.72$ & $\operatorname{Pr}=1.0$ & $\operatorname{Pr}=10$ & $\operatorname{Pr}=100$ \\
\hline 0.1 & 0.9006 & 1.0773 & 3.7373 & 12.3165 & 0.9006 & 1.0773 & 3.7370 & 12.3163 \\
1.0 & 0.8278 & 1.0000 & 3.6478 & 12.2265 & 0.8279 & 1.0000 & 3.6475 & 12.2263 \\
3.0 & 0.7140 & 0.8772 & 3.5010 & 12.0814 & 0.7143 & 0.8774 & 3.5008 & 12.0810 \\
5.0 & 0.6374 & 0.7917 & 3.3859 & 11.9694 & 0.6384 & 0.7926 & 3.3861 & 11.9689 \\
\hline
\end{tabular}

in which primes denote the differentiation with respect to $\eta, d=1 / D, \alpha=c / a$ is the unsteadiness parameter and $\operatorname{Pr}=v / \alpha_{m}$ is the Prandtle number. Further, $\lambda$ is the buoyancy or mixed convection parameter defined as $\lambda=G r_{x} / \operatorname{Re}_{x}^{2}$ where $G r_{x}=g \beta\left(T_{w}-T_{\infty}\right) x^{3} / v^{2}$ and $\operatorname{Re}_{x}=u_{w} x / v$ are the local Grashof and Reynold numbers, $D=D a_{x} \operatorname{Re}_{x}$ where $D a_{x}=K / x^{2}=$ $K_{1}(1-c t) / x^{2}$ is the local Darcy number and $K_{1}$ is the initial permeability.

The physical quantities skin friction coefficient $C_{f}$ and the local Nusselt number $N u_{x}$ are defined as

$$
\begin{gathered}
C_{f}=\frac{2 \tau_{w}}{\rho u_{w}^{2}}, \\
N u_{x}=\frac{x q_{w}}{k\left(T_{w}-T_{\infty}\right)},
\end{gathered}
$$

where the skin friction $\tau_{w}$ and the heat transfer from the sheet $q_{w}$ are given by

$$
\begin{gathered}
\tau_{w}=\mu\left(\frac{\partial u}{\partial y}\right)_{y=0}, \\
q_{w}=-k\left(\frac{\partial T}{\partial y}\right)_{y=0},
\end{gathered}
$$

with $\mu$ and $k$ being dynamic viscosity and thermal conductivity, respectively.

Using transformation on (2.7), we get

$$
\begin{aligned}
\frac{1}{2} C_{f} \operatorname{Re}_{x}^{1 / 2} & =f^{\prime \prime}(0), \\
N u \operatorname{Re}_{x}^{-1 / 2} & =-\theta^{\prime}(0) .
\end{aligned}
$$




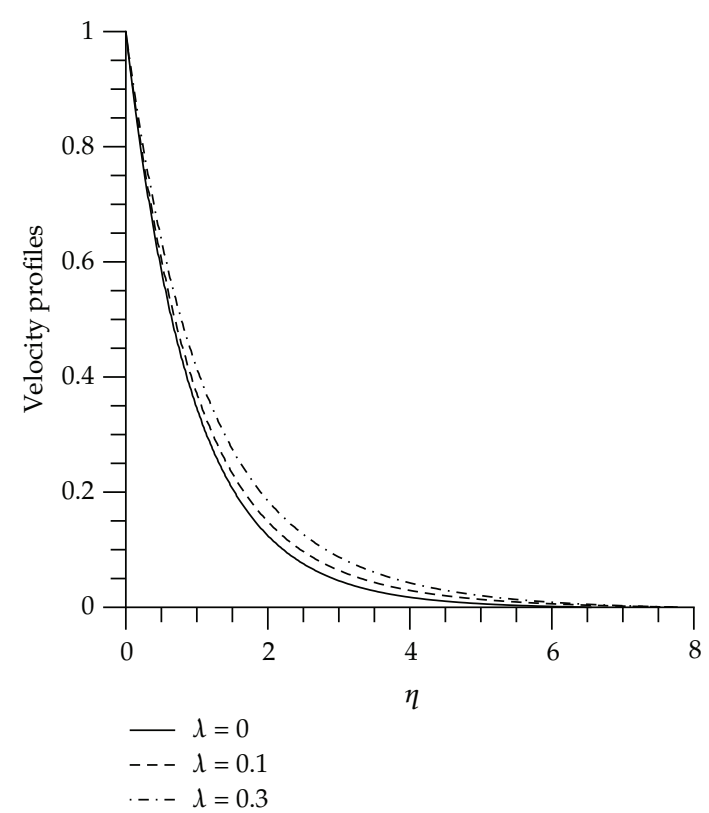

(a)

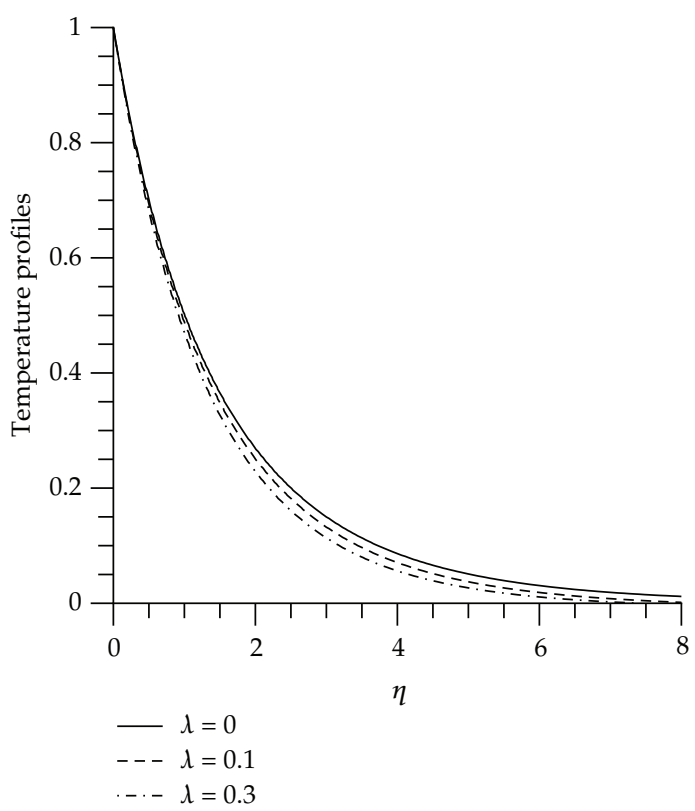

(b)

Figure 6: Effect of mixed convection parameter $\lambda$ for the case of $\alpha=d=A^{*}=B^{*}=0.1, \operatorname{Pr}=0.72$ on (a) velocity distributions $f^{\prime}(\eta)$, (b) temperature distributions $\theta(\eta)$.

\section{Solution of the Problem}

\subsection{Numerical Solution}

Equations (2.9) and (2.10) can be expressed as

$$
\begin{gathered}
f^{\prime \prime \prime}=-\left(f f^{\prime \prime}-f^{\prime 2}-\alpha\left(f^{\prime}+\frac{1}{2} \eta f^{\prime \prime}\right)+\lambda \theta-d f^{\prime}\right), \\
\theta^{\prime \prime}=-\frac{1}{\operatorname{Pr}}\left(A^{*} f^{\prime}+B^{*} \theta\right)+\operatorname{Pr}\left(f^{\prime} \theta-f \theta^{\prime}\right)+\alpha\left(2 \theta+\frac{1}{2} \eta \theta^{\prime}\right),
\end{gathered}
$$

and the corresponding boundary conditions are

$$
f(0)=0, \quad f^{\prime}(0)=1, \quad f^{\prime \prime}(0)=\alpha_{1}, \quad \theta(0)=1, \quad \theta^{\prime}(0)=\alpha_{2},
$$

where $\alpha_{1}$ and $\alpha_{2}$ are the missing initial conditions. These are determined by the shooting method in conjunction with implicit sixth order Runge-Kutta integration. The results obtained are discussed in Section 4. 


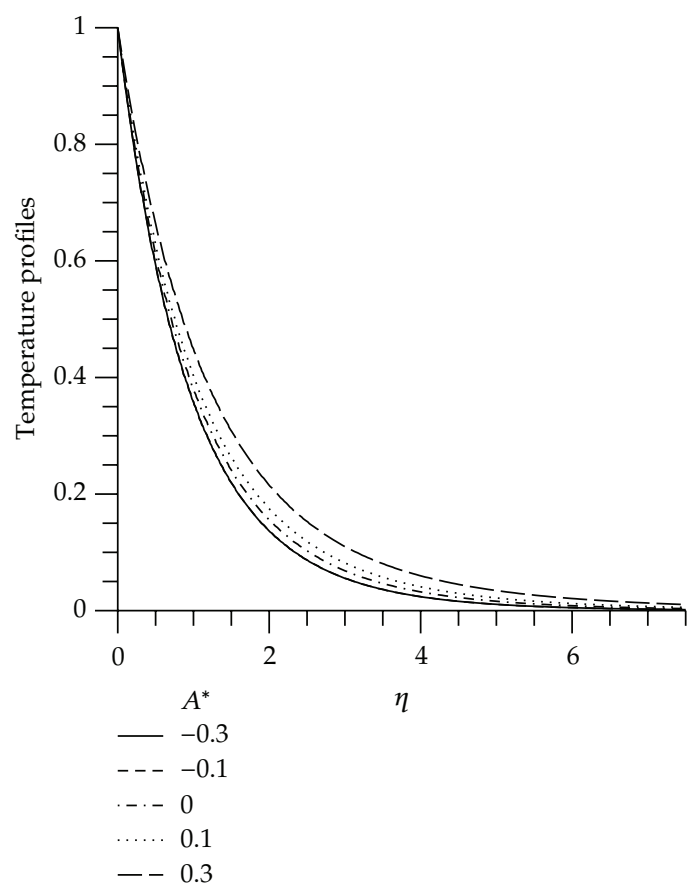

(a)

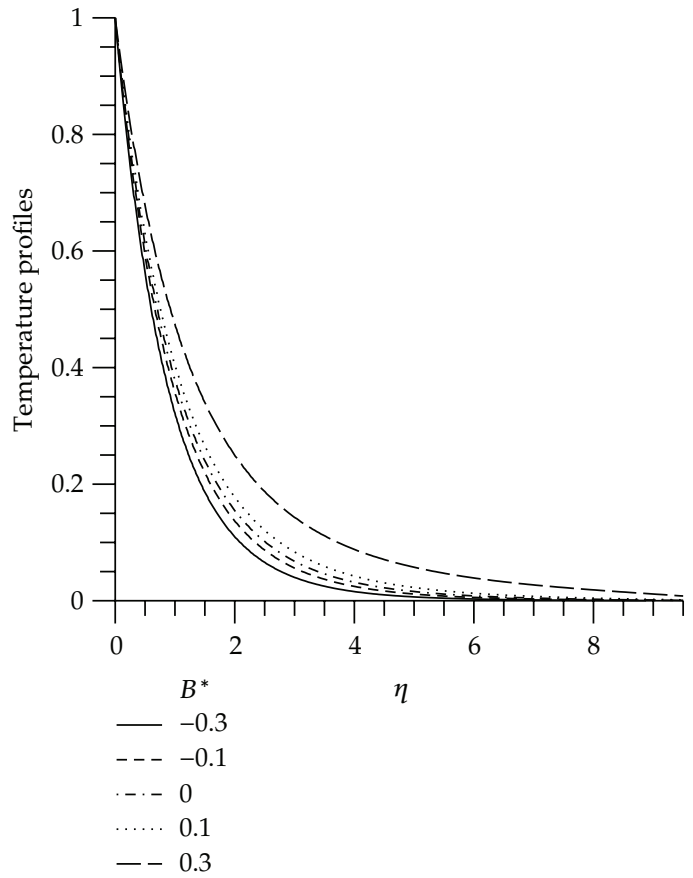

(b)

Figure 7: (a) Effect of space-dependent heat generation/absorption parameter $A^{*}$ on temperature distribution $\theta(\eta)$ for the case of $\operatorname{Pr}=0.72, \alpha=0.3, \lambda=0.1, d=0.1$ and $B^{*}=0.05$ (b) Effect of temperaturedependent heat generation/absorption parameter $B^{*}$ on temperature distribution $\theta(\eta)$ for the case of $\operatorname{Pr}=0.72, \alpha=0.3, \lambda=0.1, d=0.1$, and $B^{*}=0.05$.

\subsection{Perturbation Solution for Small Parameter $\alpha$}

We assume that both the mixed convection parameter $\lambda$ and the unsteadiness parameter $\alpha$ are small, and take $\lambda=m \varepsilon$ where $m=O(1)$ and $\alpha=\varepsilon$. Equations (2.9) and (2.10) yield

$$
\begin{gathered}
f^{\prime \prime \prime}+f f^{\prime \prime}-f^{\prime 2}-\varepsilon\left(f^{\prime}+\frac{1}{2} \eta f^{\prime \prime}\right)+m \varepsilon \theta-d f^{\prime}=0, \\
\frac{1}{\operatorname{Pr}}\left(\theta^{\prime \prime}+A^{*} f^{\prime}+B^{*} \theta\right)+f \theta^{\prime}-f^{\prime} \theta-\varepsilon\left(2 \theta+\frac{1}{2} \eta \theta^{\prime}\right)=0 .
\end{gathered}
$$

Now expanding $f$ and $\theta$ in powers of $\varepsilon$

$$
\begin{aligned}
& f(\eta)=\sum \varepsilon^{n} f_{n}(\eta), \\
& \theta(\eta)=\sum \varepsilon^{n} \theta_{n}(\eta),
\end{aligned}
$$


the zeroth order system is given by

$$
\begin{gathered}
f_{0}{ }^{\prime \prime \prime}+f_{0} f_{0}{ }^{\prime \prime}-f_{0}{ }^{\prime 2}-d f_{0}{ }^{\prime}=0 \\
\frac{1}{\operatorname{Pr}}\left(\theta_{0}{ }^{\prime \prime}+A^{*} f_{0}{ }^{\prime}+B^{*} \theta_{0}\right)+f_{0} \theta_{0}{ }^{\prime}-f_{0}{ }^{\prime} \theta_{0}=0,
\end{gathered}
$$

with

$$
\begin{gathered}
f_{0}(0)=0, \quad f_{0}{ }^{\prime}(0)=1, \quad \theta_{0}(0)=1, \\
f_{0}{ }^{\prime}(\infty)=0, \quad \theta_{0}(\infty)=0 .
\end{gathered}
$$

The exact solution of (3.5) is

$$
f_{0}(\eta)=\frac{1}{c}\left(1-e^{-c \eta}\right),
$$

where $c=\sqrt{1+d}$.

Substituting (3.8) in (3.6) and using Padé approximation, the temperature $\theta_{0}$ is

$$
\theta_{0}(\eta)=\frac{1.0+0.60 \eta+0.21 \eta^{2}}{1.0+1.23 \eta+0.71 \eta^{2}+0.22 \eta^{3}+0.04 \eta^{4}+0.008 \eta^{5}+0.001 \eta^{6}}
$$

The first order system can be expressed as

$$
\begin{gathered}
f_{1}^{\prime \prime \prime}+\frac{1}{c}\left(1-e^{-c \eta}\right) f_{1}^{\prime \prime}-\left(d+2 e^{-c \eta}\right) f_{1}^{\prime}-c e^{-c \eta} f_{1}+m \theta_{0}-e^{-c \eta}+\frac{1}{2} c \eta e^{-c \eta}=0 \\
\frac{1}{\operatorname{Pr}} \theta_{1}^{\prime \prime}+\frac{1}{c}\left(1-e^{-c \eta}\right) \theta_{1}^{\prime}+\left(\frac{B^{*}}{\operatorname{Pr}}-e^{-c \eta}\right) \theta_{1}+\left(\frac{A^{*}}{\operatorname{Pr}}-\theta_{0}\right) f_{1}{ }^{\prime}+\left(f_{1}-\frac{1}{2} \eta\right) \theta_{0}{ }^{\prime}-2 \theta_{0}=0 .
\end{gathered}
$$

The resulting expressions of $f_{1}$ and $\theta_{1}$ are

$$
\begin{gathered}
f_{1}(\eta)=0.13 \eta^{2}-0.27 \eta^{4}+0.009 \eta^{5}-0.001 \eta^{6}+0.0002 \eta^{7} \\
\theta_{1}(\eta)=\frac{-1.35 \eta+0.30 \eta^{2}}{1.0+0.31 \eta+0.07 \eta^{2}+0.03 \eta^{3}}
\end{gathered}
$$

and finally the two term perturbation solutions of (3.3) are 


$$
\begin{aligned}
& f(\eta)=f_{0}(\eta)+\varepsilon f_{1}(\eta), \\
& \theta(\eta)=\theta_{0}(\eta)+\varepsilon \theta_{1}(\eta),
\end{aligned}
$$

or

$$
\begin{aligned}
& f(\eta)=\frac{1}{c}\left(1-e^{-c \eta}\right)+\varepsilon\left(0.13 \eta^{2}-0.27 \eta^{4}+0.009 \eta^{5}-0.001 \eta^{6}+0.0002 \eta^{7}\right) \\
& \theta(\eta)= \frac{1.0+0.60 \eta+0.21 \eta^{2}}{1.0+1.23 \eta+0.71 \eta^{2}+0.22 \eta^{3}+0.04 \eta^{4}+0.008 \eta^{5}+0.001 \eta^{6}} \\
&+\varepsilon\left(\frac{-1.35 \eta+0.30 \eta^{2}}{1.0+0.31 \eta+0.07 \eta^{2}+0.03 \eta^{3}}\right)
\end{aligned}
$$

\section{Discussion}

The effects of various physical parameters on the velocity, temperature, local skin friction, and local Nusselt number are discussed. In Table 1, comparison between analytical and numerical results is presented showing a very good agreement. To compare our results with the earlier work for the steady state fluid flow, we take $\alpha=\lambda=d=A^{*}=B^{*}=0$ in (2.9). These results are compared with those given in $[7,9,11,24]$ in Table 2 . In Tables 3 and 4 , the skin friction coefficient and the Nusselt number, for various values of $\operatorname{Pr}$ and $d$, are presented and compared with [23]. The comparisons made in Tables 2-4 make a perfect match. Henceforth, the results discussed in the following paragraph are due to the shooting method (Figures 2-7).

The variation of the skin friction coefficient and the local Nusselt number are shown in Figures 2(a) and 2(b). It is observed that there is an increase in the skin friction coefficient for an assisting buoyant flow $(\lambda>0)$ and it is opposite for an opposing flow $(\lambda<0)$. This is reasonable because one would expect the velocity to increase as the buoyancy force increases and the corresponding wall shears stress to increase as well. This in turn increases the skin friction coefficient and the heat transfer rate at the surface. The unsteady effects are shown by the variation of $\alpha$ for fixed values of $\lambda=0.1, \operatorname{Pr}=0.72, d=0.1$, and $A^{*}=B^{*}=0.1$ (see Figures 3(a) and 3(b)). It is seen that the horizontal velocity and the boundary layer decreases with the increase of $\alpha$ which must be the case for decreasing wall velocity. Figures $4(a)$ and 4 (b) represent the graph of velocity and temperature profiles for different increasing values of Prandtl number Pr. It is clearly seen that the effect of the Prandtl number Pr is to decrease the temperature throughout the boundary layer resulting in the decrease of the thermal boundary layer thickness. The effects of porous medium on flow velocity and temperature are realized through the permeability parameter $(d=1 / D)$ as shown in Figures $5(a)$ and $5(b)$. It is obvious that an increase in porosity causes greater obstruction to the fluid flow, thus reducing the velocity and decreasing the temperature. It is well known that $\lambda=0$ corresponds to pure forced convection and with an increase of $\lambda$ the buoyancy force becomes stronger and the velocity profile of the fluid increases in the region near the surface of the sheet, which is evident from Figures 6(a) and 6(b). These figures also show that the fluid velocity increases while the temperature decreases with an increase of the mixed convection parameter $\lambda$. Figures $7(a)$ and $7(b)$ describe the effects of heat source on temperature profile. It is revealed that there is an increase of temperature and the thermal boundary layer with the increase of the parameters $A^{*}$ and $B^{*}$. The sink naturally has the opposite effect. 


\section{Conclusions}

The unsteady Darcy Brinkman mixed convection flow in a fluid saturated porous medium adjacent to a heated or cooled semi-infinite stretching surface in the presence of a heat source is investigated. Perturbation method with Padé approximation is used for the analytical solution and the shooting method for numerical solution reaching a good agreement between the two. A comparison is made with the earlier work to show the accuracy and reliability of our results. The effects of different parameters on the fluid flow and heat transfer characteristics are presented.

From these investigations the following conclusions are drawn.

(i) The Prandtl number Pr, permeability parameter $d$, and heat source/sink parameters $A^{*}$ and $B^{*}$ have significant effects whereas the unsteadiness parameter $\alpha$ and mixed convection parameter $\lambda$ have a little effect on the flow and temperature fields.

(ii) The skin friction coefficient increases for an assisting buoyant flow $(\lambda>0)$ and decreases for an opposing buoyant flow $(\lambda<0)$.

(iii) The horizontal velocity and the boundary layer thickness decrease as the unsteadiness parameter $\alpha$ increases.

(iv) The velocity and temperature decrease throughout the boundary layer with the increase of Prandtl number Pr.

(v) The velocity and temperature both decrease with an increase of the porosity of the medium.

(vi) The fluid velocity increases while the temperature decreases with the increase of the mixed convection parameter $\lambda$.

(vii) Temperature increases substantially with the increase of a heat source and decreases substantially for a heat sink.

All these observations are well supported by the physics and the boundary value problem at hand.

\section{Acknowledgment}

The authors gratefully acknowledge the comments to the reviewer.

\section{References}

[1] T. Altan, S. Oh, and H. Gegel, Metal Forming Fundamentals and Applications, American Society of Metal, Metals Park, Ohio, USA, 1979.

[2] E. G. Fisher, Extrusion of Plastics, John Wiley \& Sons, New York, NY, USA, 1976.

[3] B. C. Sakiadis, "Boundary-layer behavior on continuous solid surfaces: I. Boundary-layer equations for two-dimensional and axisymmetric flow," AIChE Journal, vol. 7, pp. 26-28, 1961.

[4] L. J. Crane, "Flow past a stretching plate," Zeitschrift für Angewandte Mathematik und Physik, vol. 21, no. 4 , pp. $645-647,1970$.

[5] L. E. Erickson, L. T. Fan, and V. G. Fox, "Heat and mass transfer on a moving continuous flat plate with suction or injection," Industrial and Engineering Chemistry Fundamentals, vol. 5, no. 1, pp. 19-25, 1966.

[6] P. S. Gupta and A. S. Gupta, "Heat and mass transfer on a stretching sheet with suction or blowing," The Canadian Journal of Chemical Engineering, vol. 55, pp. 744-746, 1977. 
[7] L. J. Grubka and K. M. Bobba, "Heat transfer characteristics of a continuous stretching surface with variable temperature," Journal of Heat Transfer, vol. 107, no. 1, pp. 248-250, 1985.

[8] C. K. Chen and M. I. Char, "Heat transfer of a continuous, stretching surface with suction or blowing," Journal of Mathematical Analysis and Applications, vol. 135, no. 2, pp. 568-580, 1988.

[9] M. E. Ali, "Heat transfer characteristics of a continuous stretching surface," Heat Mass Transfer, vol. 29, no. 4, pp. 227-234, 1994.

[10] C. H. Chen, "Laminar mixed convection adjacent to vertical, continuously stretching sheets," Heat and Mass Transfer, vol. 33, no. 5-6, pp. 471-476, 1998.

[11] E. M. A. Elbashbeshy, "Heat transfer over a stretching surface with variable surface heat flux," Journal of Physics D, vol. 31, no. 16, pp. 1951-1954, 1998.

[12] A. Ishak, R. Nazar, and I. Pop, "MHD boundary-layer flow due to a moving extensible surface," Journal of Engineering Mathematics, vol. 62, no. 1, pp. 23-33, 2008.

[13] C. Y. Wang, "Analysis of viscous flow due to a stretching sheet with surface slip and suction," Nonlinear Analysis: Real World Applications, vol. 10, no. 1, pp. 375-380, 2009.

[14] T. Hayat, Z. Abbas, and T. Javed, "Mixed convection flow of a micropolar fluid over a non-linearly stretching sheet," Physics Letters, Section A, vol. 372, no. 5, pp. 637-647, 2008.

[15] T. Hayat, M. Sajid, and I. Pop, "Three-dimensional flow over a stretching surface in a viscoelastic fluid," Nonlinear Analysis: Real World Applications, vol. 9, no. 4, pp. 1811-1822, 2008.

[16] S. Awang Kechil and I. Hashim, "Series solution of flow over nonlinearly stretching sheet with chemical reaction and magnetic field," Physics Letters, Section A, vol. 372, no. 13, pp. 2258-2263, 2008.

[17] C. Y. Wang, "Liquid film on an unsteady stretching surface," Quarterly of Applied Mathematics, vol. 48, no. 4, pp. 601-610, 1990.

[18] H. I. Andersson, J. B. Aarseth, N. Braud, and B. S. Dandapat, "Flow of a power-law fluid film on an unsteady stretching surface," Journal of Non-Newtonian Fluid Mechanics, vol. 62, no. 1, pp. 1-8, 1996.

[19] I. Pop and T. Y. Na, “Unsteady flow past a stretching sheet," Mechanics Research Communications, vol. 23, no. 4, pp. 413-422, 1996.

[20] J. B. Aarseth and J. B. Aarseth, "Heat transfer in a liquid film on an unsteady stretching surface," International Journal of Heat and Mass Transfer, vol. 43, no. 1, pp. 69-74, 1999.

[21] E. M. A. Elbashbeshy and M. A. A. Bazid, "Heat transfer over an unsteady stretching surface," Heat and Mass Transfer, vol. 41, no. 1, pp. 1-4, 2004.

[22] E. M. A. Elbashbeshy and M. A. A. Bazid, "Heat transfer over an unsteady stretching surface with internal heat generation," Applied Mathematics and Computation, vol. 138, no. 2-3, pp. 239-245, 2003.

[23] A. Ishak, R. Nazar, and I. Pop, "Steady and unsteady boundary layers due to a stretching vertical sheet in a porous medium using darcy-brinkman equation model," International Journal of Applied Mechanics and Engineering, vol. 11, no. 3, pp. 623-637, 2006.

[24] A. Ishak, R. Nazar, and I. Pop, “Heat transfer over an unsteady stretching permeable surface with prescribed wall temperature," Nonlinear Analysis: Real World Applications, vol. 10, no. 5, pp. 2909-2913, 2009.

[25] T. Hayat, M. Qasim, and Z. Abbas, "Homotopy solution for the unsteady three-dimensional MHD flow and mass transfer in a porous space," Communications in Nonlinear Science and Numerical Simulation, vol. 15, no. 9, pp. 2375-2387, 2010.

[26] T. Fang, J. Zhang, and S. Yao, "A new family of unsteady boundary layers over a stretching surface," Applied Mathematics and Computation, vol. 217, no. 8, pp. 3747-3755, 2010.

[27] S. Nadeem, A. Hussain, and M. Khan, "HAM solutions for boundary layer flow in the region of the stagnation point towards a stretching sheet," Communications in Nonlinear Science and Numerical Simulation, vol. 15, no. 3, pp. 475-481, 2010.

[28] D. A. Nield and A. Bejan, Convection in Porous Media, Springer, New York, NY, USA, 3rd edition, 2006.

[29] I. Pop and D. B. Ingham, Convective Heat Transfer: Mathematical and Computational Modeling of Viscous Fluids and Porous Media, Pergamon, Oxford, UK, 2001.

[30] D. B. Ingham and I. Pop, Eds., Transport Phenomena in Porous Media, vol. 2, Elsevier Science, 2002.

[31] A. Bejan, I. Dincer, S. Lorente, A. F. Miguel, and A. H. Reis, Porous and Complex Flow Structures in Modern Technologies, Springer, New York, NY, USA, 2004.

[32] D. B. Ingham and I. Pop, Eds., Transport Phenomena in Porous Media, vol. 3, Elsevier, Oxford, UK, 2005.

[33] D. B. Ingham, A. Bejan, E. Mamut, and I. Pop, Eds., Emerging Technologies and Techniques in Porous Media, Kluwer, Dordrecht, The Netherlands, 2004.

[34] K. Vafai, Ed., Handbook of Porous Media, Marcel Dekker, New York, NY, USA, 2000.

[35] K. Vafai, Handbook of Porous Media, Taylor and Francis, New York, NY, USA, 2nd edition, 2005. 
[36] T. S. Chen and F. A. Strobel, "Buoyancy effects on heat and mass transfer in boundary layer on a continuous moving horizontal plate," Numerical Heat Transfer, vol. 3, no. 1, pp. 115-130, 1980.

[37] F. A. Strobel and T. S. Chen, "Buoyancy effects on heat and mass transfer in boundary layers adjacent to inclined continuous moving sheets," Numerical Heat Transfer, vol. 3, no. 4, pp. 461-481, 1980.

[38] A. Moutsoglou and T. S. Chen, "Buoyancy effects in boundary layers on inclined, continuous, moving sheets," Journal of Heat Transfer, vol. 102, no. 2, pp. 371-373, 1980.

[39] J. R. Fan, J. M. Shi, and X. Z. Xu, "Similarity solution of mixed convection over a horizontal moving plate," Heat and Mass Transfer, vol. 32, no. 3, pp. 199-206, 1997.

[40] C. H. Chen, "Laminar mixed convection adjacent to vertical, continuously stretching sheets," Heat and Mass Transfer, vol. 33, no. 5-6, pp. 471-476, 1998.

[41] M. Ali and F. Al-Yousef, "Laminar mixed convection from a continuously moving vertical surface with suction or injection," Heat and Mass Transfer, vol. 33, no. 4, pp. 301-306, 1998.

[42] C. H. Chen, "Mixed convection cooling of a heated, continuously stretching surface," Heat Mass Transfer, vol. 36, no. 1, pp. 79-86, 2000.

[43] M. Ali and F. Al-Yousef, "Laminar mixed convection boundary layers induced by a linearly stretching permeable surface," International Journal of Heat and Mass Transfer, vol. 45, no. 21, pp. 4241-4250, 2002.

[44] K. Vajravelu and A. Hadjinicolaou, "Heat transfer in a viscous fluid over a stretching sheet with viscous dissipation and internal heat generation," International Communications in Heat and Mass Transfer, vol. 20, no. 3, pp. 417-430, 1993. 


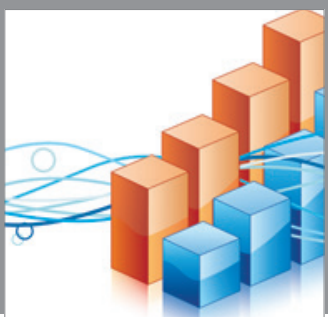

Advances in

Operations Research

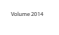

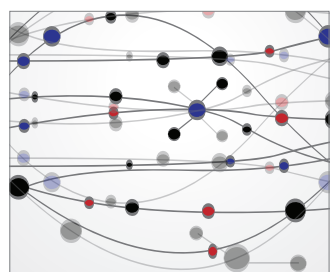

\section{The Scientific} World Journal
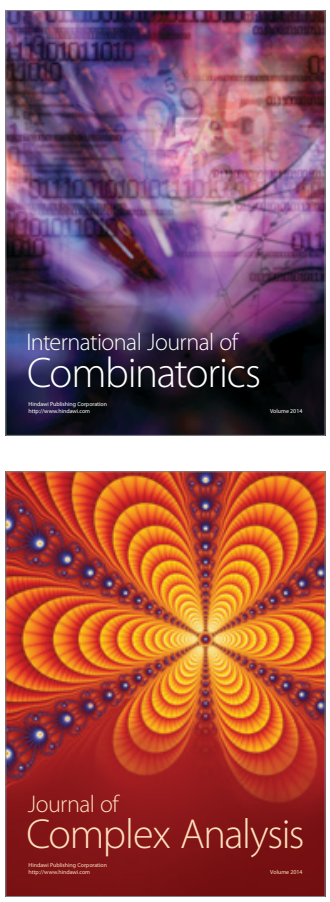

International Journal of

Mathematics and

Mathematical

Sciences
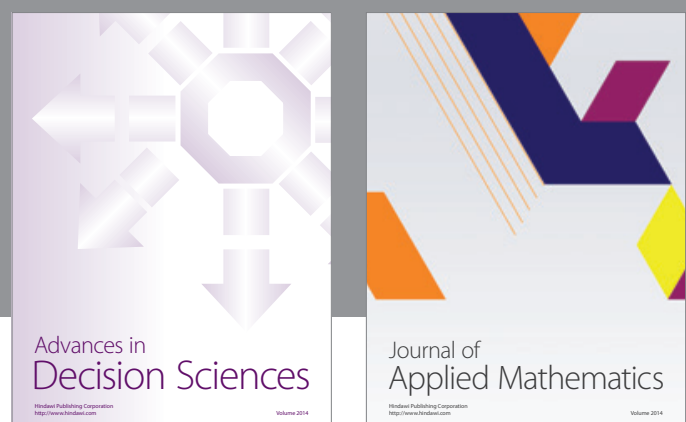

Journal of

Applied Mathematics
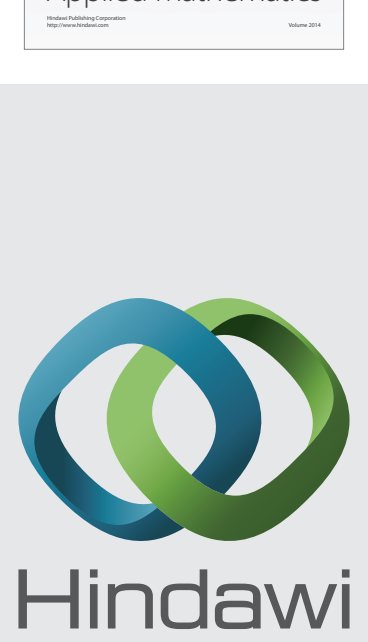

Submit your manuscripts at http://www.hindawi.com
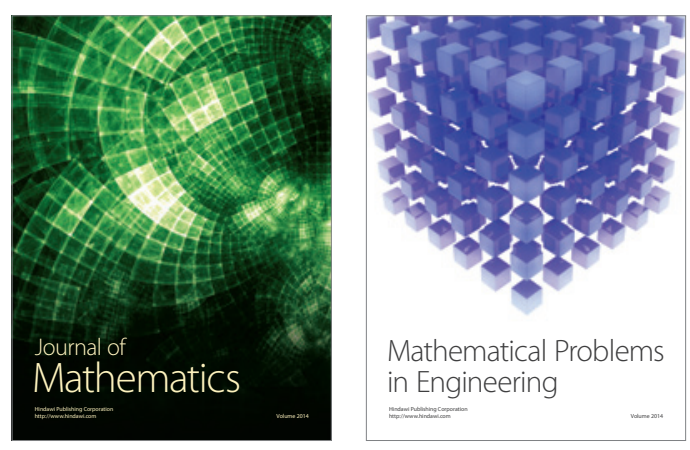

Mathematical Problems in Engineering
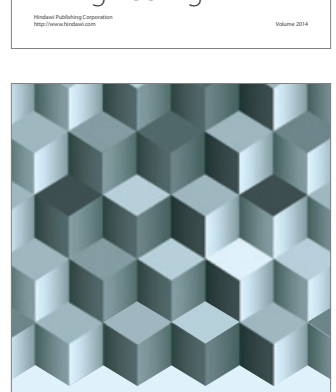

Journal of

Function Spaces
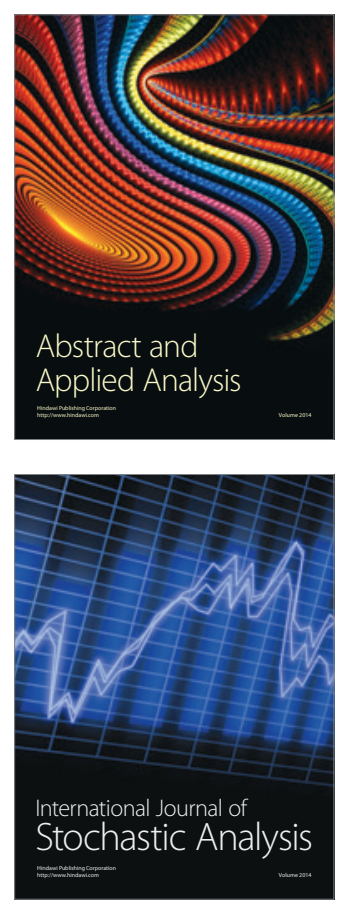

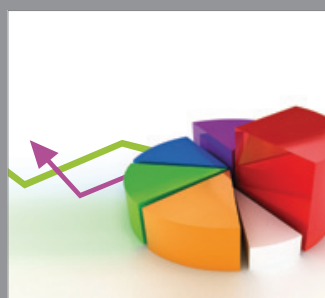

ournal of

Probability and Statistics

Promensencen
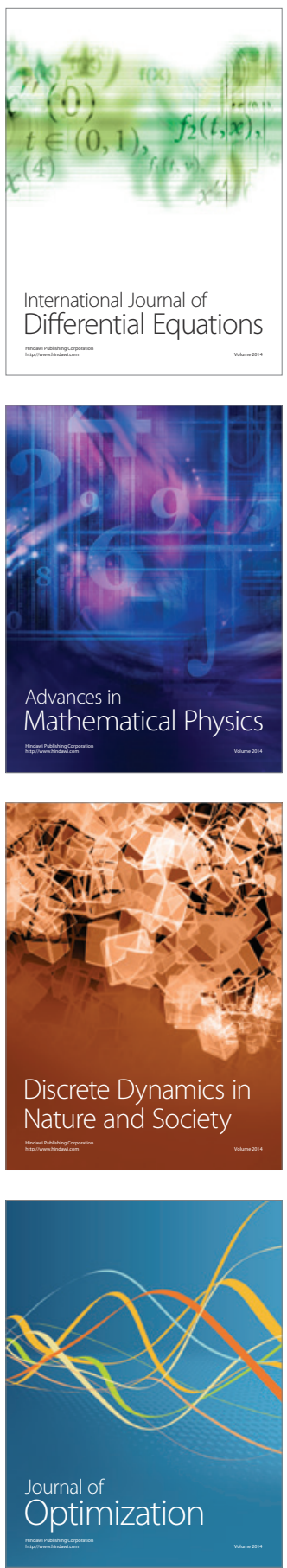